\title{
Review Article \\ Experimental Rat and Mouse Carotid Artery Surgery: Injury and Remodeling Studies
}

\author{
Andrew W. Holt and David A. Tulis \\ Department of Physiology, Brody School of Medicine, East Carolina University, Room 6E-108, Greenville, NC 27834, USA \\ Correspondence should be addressed to David A. Tulis; tulisd@ecu.edu
}

Received 20 February 2013; Accepted 31 March 2013

Academic Editors: M. Barczyński and Y. Izci

Copyright ( 2013 A. W. Holt and D. A. Tulis. This is an open access article distributed under the Creative Commons Attribution License, which permits unrestricted use, distribution, and reproduction in any medium, provided the original work is properly cited.

In cardiovascular research, translation of benchtop findings to the whole body environment is often critical in order to gain a more thorough and comprehensive clinical evaluation of the data with direct extrapolation to the human condition. In particular, developmental and/or pathophysiologic vascular growth studies often employ in vitro approaches such as cultured cells or tissue explant models in order to analyze specific cellular, molecular, genetic, and/or biochemical signaling factors under pristine controlled conditions. However, validation of in vitro data in a whole body setting complete with neural, endocrine, and other systemic contributions provides an essential proof of concept from a clinical perspective. Several well-characterized experimental in vivo models exist that provide excellent proof-of-concept tools to examine vascular growth and remodeling in the whole body. This paper will examine the rat carotid artery balloon injury model, the mouse carotid artery wire denudation injury model, and rat and mouse carotid artery ligation models with particular emphasis on minimally invasive surgical access to the site of intervention. Discussion will include key scientific and technical details as well as caveats, limitations, and considerations for the practical use of each of these valuable experimental models.

\section{Introduction}

Translation of basic research discoveries to clinical efficacy and utility requires experimental models that encompass the whole body environment complete with neural, hormonal, endocrine, and other systemic contributions from associated cell and tissue types. All of these factors potentially influence the outcomes witnessed when using in vitro preparations and single cell or isolated tissue model systems. Particularly regarding vascular growth and remodeling studies, numerous experimental animal models in a variety of vascular beds have been used over the years as proof-of-concept in vivo approaches. Considering the carotid artery as a model system, several rodent-based approaches are widely used and accepted as clinically relevant in terms of examining the in vivo responses to injury-induced growth and remodeling. The rat carotid artery balloon injury model utilizes mechanical damage caused by a balloon catheter which results in mural distension and removal of the intimal endothelial lining. The mouse wire injury model uses an angiocatheter guide wire to remove the endothelial lining and denude the vessel in the absence of medial wall distension. Ligation experiments, using the common carotid artery or one of its branches, result in full or partial flow obstruction with resultant hemodynamic perturbations. All of these models elicit reproducible vessel growth and remodeling responses characterized by vascular smooth muscle (VSM) cell dedifferentiation and alteration into a synthetic and proliferative phenotype concomitant with recruitment of resident and circulating cells and endothelial cell mitogenesis. These multifactorial growth processes eventuate in the establishment of a stenotic neointima in time-dependent fashion. These models then provide valuable approaches to analyze growth and remodeling under in vivo conditions that can be used to validate and substantiate in vitro results.

The rat carotid artery balloon injury model, originally described by Clowes and colleagues [1-4] and subsequently comprehensively characterized by our lab $[5,6]$ and others, has been utilized in a wide variety of basic and clinical science research projects. In brief, this approach involves 
surgical access to the common carotid artery and carotid artery bifurcation, isolation of a segment of the vasculature including the external carotid branch, creation of an arteriotomy on the external carotid for luminal intervention, and insertion of a balloon catheter into the common carotid to elicit the injury. The adaptive response to this injury involves VSM cell proliferation, migration, and apoptosis, partial endothelial cell regeneration, medial and adventitial thickening, enhanced neointimal and medial fibrosis, and establishment of an invasive neointima.

The mouse wire denudation injury model was developed by Lindner et al. [7], Sullivan Jr. et al., and Iafrati et al. $[8,9]$ and consists of carotid artery access and isolation, establishment of an external carotid arteriotomy, and insertion of an angiocatheter guide wire to elicit endothelial denudation and removal of the intimal layer through the length of the common carotid artery in the absence of medial wall distension. The growth response following this injury is characterized by rapid platelet adherence to the subendothelial matrix and intimal and medial VSM cell proliferation and migration in the presence of patent arterial flow, eventuating in a concentricneointima by 2 to 4 weeks. Repair of the endothelial lining is normally completed by 4 weeks after the injury due to endothelial replication and migration from the border zones.

Several distinct carotid artery ligation models also exist that elicit varying degrees of flow alteration and arterial remodeling. Ligation of the common carotid artery ceases blood flow through the common carotid and downstream branches and eventuates in robust medial VSM cell proliferation and neointimal and medial wall growth with reduced vessel caliber in the presence of intact endothelium [10, 11]. Ligation of the external carotid artery branch significantly reduces arterial flow through the common carotid artery and results in flow-mediated reduction in lumen diameter and medial wall mass along with decreased VSM cell replication and elastin content $[12,13]$. Combined, these three models and their variations permit in vivo analyses of injury-induced vessel growth and remodeling from mural distension and endothelial removal, from endothelial denudation without vascular distension, or from flow-mediated changes and hemodynamic alterations in the face of an uncompromised vessel wall.

All of these procedures require carotid artery vascular access as an initial component of their minimally invasive interventions. Although "vascular access" has historically referred to luminal catheterization for purposes of delivering therapeutic agents such as chemotherapy or antibiotics or for blood transfusions, a broader definition of "vascular access" is surgical intervention for purposes of exposure and isolation of a vessel segment for subsequent intervention and/or manipulation. In this light, these experimental models require vascular access of the common carotid artery and/or their internal and external arterial branches in order for subsequent vessel injury or ligature installation. This paper focuses on minimally invasive surgical aspects of vascular access and experimental protocols for rat and mouse carotid artery injury and ligation models.

\section{Materials and Methods}

We have recently published comprehensive, step-by-step experimental protocols and details for the rat carotid artery balloon injury model [5] along with associated histomorphometric analyses for rat and mouse arterial injury studies [6]. The reader is advised to consult these works for expanded discussion of these methodologies.

2.1. Animals. All animal care and experimental procedures must adhere strictly to the recommendations of the Guide for Care and Use of Laboratory Animals (DHEW, NIH) Ref. number 85-23 (revised 1996) and the Public Health Service Policy on Humane Care and Use of Laboratory Animals (revised 2002), as well to the guidelines of the local Institutional Animal Care and Use Committee. For the rat models, a variety of strains has been utilized over the years with the most common strain being Sprague-Dawley (Harlan). Male rats are preferred due to the potential impact of hormones on cellular function that could impact growth responses [11]. Also, varied animal body weights have been used, but it is critical that a narrow range of weights are used (suggested a range of 350-450 grams) within any particular study to avoid inherent variation in basal vessel caliber. Our experiments generally use a range of 425-475 grams body weight. Unless experimental protocol dictates otherwise, rats are kept on standard rodent chow and water ad libitum perioperatively.

For the mouse models, a variety of background strains has been used per specific experimental strategies, and body weights generally range from $\sim 18$ to 25 grams (20-25 weeks of age), with certain protocols using mice up to 30 grams body weight. Again, keeping the body weight within a narrow range minimizes inherent variation caused by differences in basal vessel caliber. Unless otherwise needed, mice are kept on standard rodent chow and water ad libitum. Lastly, even though a vast majority of these surgeries are performed on the left carotid artery, the right carotid artery can be used as well. However, the left carotid artery presents a longer section for intervention and is the vessel of choice for this author and many other investigators. If one chooses to use the right carotid artery, keep in mind that it branches off the innominate (brachiocephalic) artery after bifurcation from the aortic root and so presents with a shorter vessel segment for intervention. Also, the proximal innominate artery is not suitable for manipulation (as an extension of the right common carotid) due to larger resting caliber.

2.2. Aseptic and Sterile Practices. Per institutional guidelines, sterile instruments and solutions and a sterile surgical field and surgical attire (scrubs, gloves, facemasks, head and hair covering, and/or surgical gown) are required for survival surgery. Sterilization is commonly performed on these items using an autoclave, gas (ethylene oxide), dry heat via a glass bead sterilizer, and/or chemical treatment. Aseptic technique using sterile instruments and practice is then used for all survival surgeries.

2.3. Vascular Access. Following sedation to a surgical plane, rats or mice are laid supine on a sterile surface, legs are gently 
retracted, the neck area is shaved and cleansed with alternating skin disinfectant (i.e., chlorhexidine) and ethanol scrubs, and the surgical area (neck) aseptically draped to help prevent contamination at the surgical site. Using sterile sharp/blunt serrated-edge scissors, a straight incision is made centrally in the neck region from just below the chin to the top of the sternum just above the ribcage. Using sterile medium hemostats and/or dull forceps, the skin on both sides of the incision is separated and blunt-dissected and the underlying tissue dissected away from the skin circumferentially around the entire incision wound. Next, using sterile medium sharp/sharp scissors, the fascia overlying the glandular tissue is separated to expose the underlying glands, which are then gently dissected apart via blunt dissection to expose the muscular layer. As most carotid artery surgeries use the left carotid artery, the longitudinal left aspect of the central and adjacent muscular tissues is blunt-dissected apart and retracted. With continued blunt dissection, the left common carotid artery and the distal aspect of the carotid artery cephalic to the internal and external bifurcation are isolated and made free of overlying fascia and adjacent nerves. Keep in mind that the vagus nerve resides immediately adjacent to and sometimes on top of the carotid vasculature and so careful practice should be made to avoid manipulation of this and all associated nerves (including the carotid body nerve plexus at the bifurcation). Once the vessel segments are isolated and freed of fascia, the appropriate placement of sutures will depend on the desired method of vascular intervention. For the rat carotid artery balloon injury and mouse wire injury models, three sterile sutures are placed around the common and external carotid artery branch in order to isolate a segment for vascular intervention (see Figure 1): one suture proximal is on the common carotid (No. 1 in Figure 1), one is on the external carotid branch immediately distal to the bifurcation and loosely looped (No. 2 in Figure 1), and one is as distal as possible on the external branch and loosely looped with one long end used for retraction (No. 3 in Figure 1). Also, if arterial branching (including ascending pharyngeal, occipital, and superior thyroid arteries) off the external carotid artery is observed and is readily accessible, these should be ligated using sterile suture in order to avoid retrograde flow. If conducting carotid artery ligation/flow disruption models, placement of these sutures may differ from those depicted in Figure 1. In fact, in the ligation models, placement and ligature of a single or multiple sutures is the cause for the growth stimulus, whereas in the balloon injury and wire injury models, the ligations are used simply to control blood flow in order to perform the injury. Whether the balloon injury, wire injury, or ligation models are used, at this point, all sutures should be set and untied. Throughout all procedures, the level of sedation of the animal should be routinely checked, and all exposed tissues should be kept moist using sterile saline or sterile solution of choice.

2.4. Rat Carotid Artery Balloon Injury Model. The most distal suture on the external branch (No. 3 in Figure 1) should be tied first using a double knot, and the long end of this suture should be retracted cephalically. Next, using the

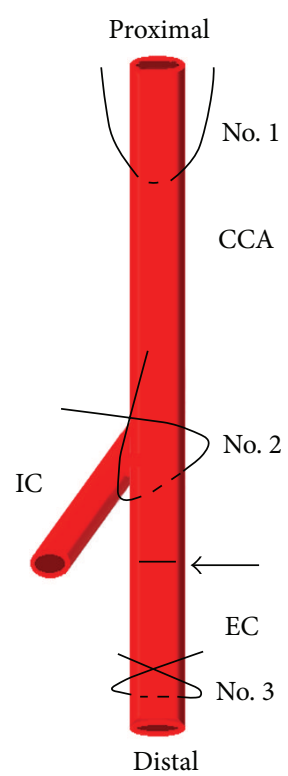

Figure 1: Simplified schematic showing carotid artery vasculature and sites for placement of arterial sutures for retraction and/or ligation. During vascular access, suture No. 1 is looped around the most proximal portion of the left common carotid artery (CCA), suture No. 2 is looped around the external branch immediately distal to the internal carotid (IC) and external carotid (EC) bifurcation, and suture No. 3 is looped around the EC artery as distally as possible. The arteriotomy site for insertion of the balloon catheter is shown on the EC branch between the bifurcation and the site of distal ligature (No. 3) and is indicated by an arrow and straight line.

suture on the common carotid artery (No. 1 in Figure 1), gently "tent up" the vessel and place an arterial clamp on the common carotid artery as proximally as possible. All blood flow (minus potential retrograde flow via the internal carotid artery branch or other unligated branches) should be stopped at this point. Isolating a section on the external carotid artery and using sterile small microscissors, perform a transverse arteriotomy on the external branch (see arrow in Figure 1) and carefully maintain hemostasis. If desired with the assistance of a guiding trocar (a sterile 18-gauge ultrathin walled needle), insert uninflated 2 French arterial balloon embolectomy catheters (Edwards Lifesciences) through the arteriotomy and down the entire length of the common carotid artery to the aortic arch (obviously at this point the clamp on the common carotid has been removed). Slowly inflate the balloon to a predetermined volume (suggested $0.02 \mathrm{~mL})$ or pressure ( 2.0 atm) manually (via syringe) or with the use of a perfuse-inflation device and barometer. Clamp the syringe (via stopcock) or set the inflation device to maintain balloon inflation pressure and withdraw the inflated balloon distally with rotation along the entire length of the common carotid artery to just immediately proximal to the bifurcation. Deflate the balloon and repeat this procedure two more times for a total of three passages of an inflated balloon through the common carotid to ensure complete and reproducible removal of the endothelial lining and nonbiased distension of the vessel wall. Following the last passage of 
the balloon, deflate and withdraw the balloon through the arteriotomy and immediately tie off the last remaining suture two times (No. 2 in Figure 1) that had been looped around the external branch but not tied up to this point to ensure adequate hemostasis. Check the common carotid artery for pulsatility and patent blood flow and inspect all vasculature for bleeding paying particular attention to the external branch and the site of the arteriotomy. Following adequate hemostasis, remove all remaining sutures. Place overlying tissues on top of the carotid vasculature in layers; close and suture glandular tissue using sterile suture making sure to double-knot and tuck both ends. Close the skin incision using the method of choice (we find sterile skin staples as the best option), swab with an antiseptic/bactericide agent if desired, and continue with postoperative care as needed. Pertinent to a specific research strategy, plan the next series of experiments accordingly to obtain relevant vascular tissues for use in histology, expression analyses, or other endpoint analyses as desired.

2.5. Mouse Carotid Artery Wire Denudation Injury Model. Following vascular access and establishment of an external carotid arteriotomy, with or without use of a surgical stereomicroscope (based on surgeon's experience), an angiocatheter guide wire $(\sim 0.35 \mathrm{~mm}$ diameter $)$ is inserted through the arteriotomy site, advanced down the length of the common carotid artery to the aortic arch, and then withdrawn thrice with rotation to efficiently and circumferentially remove the endothelial lining along the entire length of the common carotid artery. The wire is removed, and the suture on the external branch is tied twice to ensure hemostasis. All untied sutures are removed, and the tissues and skin closed in layers as described for the rat model.

2.6. Rat and Mouse Carotid Artery Ligation Models. Following vascular access and isolation of the common carotid artery and its branches as described above, full or partial flow obstruction can be made depending on the site of ligature placement. Ligation of the common carotid artery immediately proximal to the bifurcation results in complete blockage and stagnation of carotid artery blood flow as described in [11]. Alternately, one may choose to ligate only the external carotid artery branch just distal to the bifurcation, thereby reducing common carotid artery blood flow to internal carotid artery levels $[12,13]$. Lastly, although not a focus of this paper, if an investigator chooses to ligate one or several of the smaller vessels that branch off the external carotid artery such as the ascending pharyngeal, occipital, and/or superior thyroid arteries, only those vessels of interest should be ligated at this point to induce flow obstruction [15].

2.7. Histology and Endpoint Analyses. Per specific requirements of any particular experimental strategy, endpoint analyses for carotid artery injury studies commonly include short time points ( $\leq 72$ hours) on fresh, snap-frozen, or perfusion-fixed tissues for evaluation of mechanistic factors that contribute to growth adaptations and longer time points (generally 2 weeks for rats; 2-4 weeks for mice) for evaluation of growth-specific remodeling parameters relevant to the nature of the intervention. As we have recently described in detail [6], histomorphometry can be performed on vascular sections following establishment of these models in order to obtain indices of arterial growth and remodeling such as lumen area, perimeters of the internal and external elastic laminae (to estimate vessel expansion and changes in adaptive vessel caliber), and area and thickness of the neointima, medial wall, and adventitia.

Several common histological approaches for use in rodent vascular injury and growth studies are hematoxylin and eosin (H\&E) and Verhoeff-Van Gieson staining. Regarding $\mathrm{H} \& \mathrm{E}$, hematoxylin stains nucleic acids blue/black, while eosin stains collagen pink and cellular cytoplasm red. In brief, perfusion-fixed tissues are deparaffinized and rehydrated and are stained with a hematoxylin of choice (Mayer's 10-20 min; Ehrlich's 20-45 min), rinsed in tap water until sections colordifferentiate and turn blue ( $\leq 5 \mathrm{~min}$ ), further differentiated in $1 \%$ acid alcohol ( $1 \% \mathrm{HCl}$ in $70 \%$ ethanol) for $5-10 \mathrm{sec}$, rinsed in tap water again, then stained in $1 \%$ eosin for $\sim 10 \mathrm{~min}$, rinsed, dehydrated, and cover-slipped. Results from $\mathrm{H} \& \mathrm{E}$ staining are blue/black nuclei, red/pink cytoplasm, deep pink muscle fibers and fibrin, and red/orange red blood cells. Verhoeff-Van Gieson staining uses a combination of ferric chloride hematoxylin with an iron mordant along with an acid fuchsin/picric acid solution for elastin and collagen staining. In brief, deparaffinized and rehydrated tissues are stained in Verhoeff's solution (4\% alcoholic hematoxylin $(20 \mathrm{~mL}), 10 \%$ aqueous ferric chloride $(8 \mathrm{~mL}), 2$ grams of Lugol's iodine, 4 grams of potassium iodine, and $100 \mathrm{~mL}$ distilled water $(8 \mathrm{~mL}))$, washed in warm tap water and differentiated in $2 \%$ ferric chloride, washed and counterstained in Van Gieson solution (1\% aqueous solution of acid fuchsin $(10 \mathrm{~mL})$, saturated aqueous solution of picric acid $(200 \mathrm{~mL}))$, and dehydrated. Results from Verhoeff-Van Gieson staining are intensely blue/black elastic fibers, blue-to-black nuclei (based on variables for hematoxylin staining), and red collagen with fat and nerves and associated tissues staining yellow. Representative photomicrographs of H\&E- and Verhoeff-Van Gieson-stained rat and mouse carotid artery cross-sections are shown in Figures 2, 3, and 4.

\section{Results}

3.1. Rat Carotid Artery Balloon Injury Model. Histopathological assessment of neointimal development and vascular growth and remodeling is generally obtained 2 weeks following the injury in the rat balloon model. Figure 2 shows the representative whole artery cross-sections following Verhoeff-Van Gieson histological staining on rat uninjured (a) or balloon-injured (c) left common carotid arteries. In this staining procedure, the Verhoeff component stains elastin fibers black (primarily in the elastic laminae), and Van Gieson stain is specific for collagen and colors it red (primarily the adventitia). In the uninjured vessel (a), a patent lumen (L) and a single cell layer thick intima (arrow) are observed with a VSM-rich medial wall layer and collagenrich adventitial tissue. In the injured artery (c), a significantly reduced stenotic lumen and a robust concentric neointima 


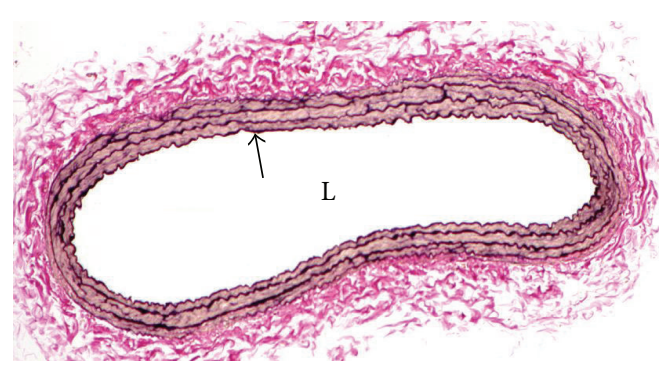

(a)

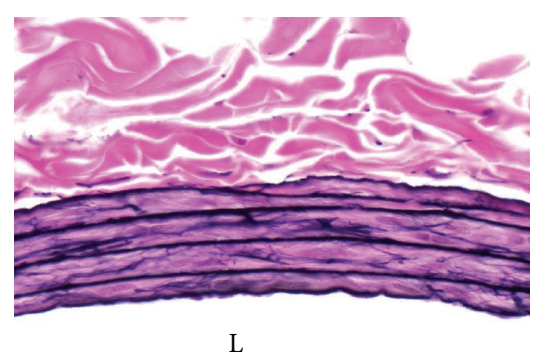

(b)

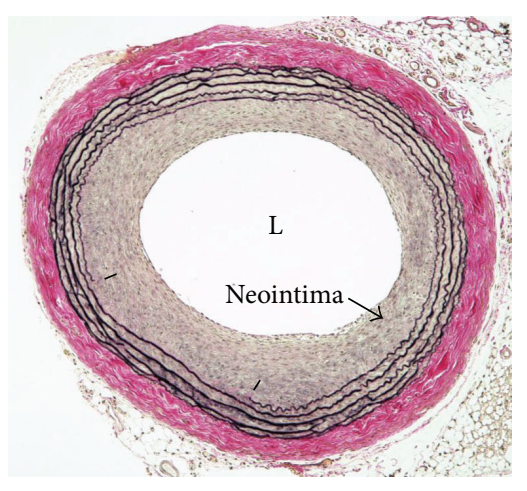

(c)

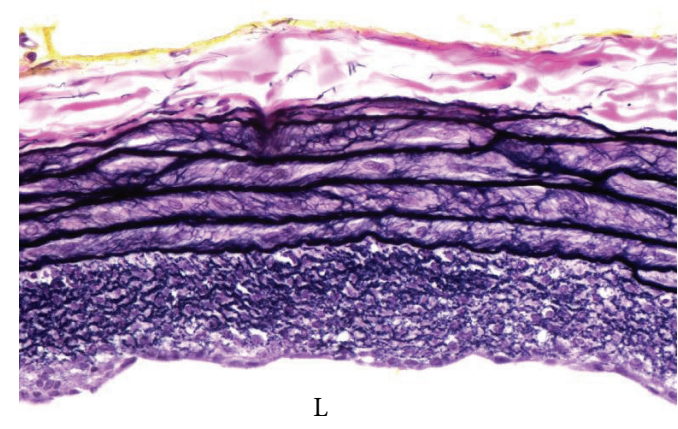

(d)

Figure 2: Representative Verhoeff-Van Gieson-stained cross-sections of rat left common carotid arteries. (a) A whole cross-section of an uninjured artery is shown with a patent lumen (L), a single cell layer thick intima (arrow), a VSM-rich medial wall, and a collagen-rich adventitia. (b) A higher magnification photo of an uninjured artery with expanded details is shown. (c) At 2 weeks after balloon injury an injured artery is shown with a significantly reduced lumen and a robust concentric neointima. (d) A higher magnification photo of an injured artery obtained 2 weeks after the injury clearly showing an elastin-rich neointima and enhanced medial wall elastin content. In all of these photomicrographs elastin fibers stained black (including the elastic laminae), and collagen and associated matrix components stained red (primarily the adventitia).

are readily observed. Additionally, in this photomicrograph, a partially ruptured internal elastic lamina is noted (lower left neointima-internal elastic lamina margin denoted by hash marks) along with a thickened and compacted collagen-rich adventitia. Figures 2(b) and 2(d) are higher magnification images of arterial cross-sections (from different animals) showing details of differential Verhoeff-Van Gieson staining and highlighting an elastin-rich neointima (Figure 2(d)).

\subsection{Mouse Carotid Artery Wire Denudation Injury Model.}

Tissues from this surgery are generally obtained between 2 and 4 weeks after the injury depending on the nature of the data evaluation. Figure 3 shows mouse artery cross-sections with/without carotid artery wire denudation: Figure 3(a) shows a hematoxylin and eosin (H\&E)-stained high magnification image of an uninjured artery with clear nuclear staining of intimal endothelial cells (arrows) and a patent lumen (L); Figure 3(b) shows an H\&E-stained high magnification image 30 minutes after the wire denudation injury, and a platelet-rich monolayer covering the intimal lining is clearly evident (arrows) along the luminal border; Figure 3(c) shows an H\&E-stained whole artery cross-section of an uninjured carotid artery; Figure 3(d) similarly shows an
H\&E-stained cross-section 4 weeks after the injury with a robust and concentric neointima and severe luminal obstruction; Figure 3(e) shows a high magnification Verhoeff-Van Gieson-stained cross-section of a sham-operated artery 4 weeks post-sham surgery with a clear lumen and a thin intimal lining; and Figure 3(f) shows a Verhoeff-Van Giesonstained cross-section 4 weeks after the injury with significant neointimal development and a partially thrombosed, plateletrich lumen (denoted by $*$ ).

3.3. Ligation Models. Rat and mouse ligation models can partially or fully obstruct proximal blood flow and resultant hemodynamic forces depending on the nature and site of ligature placement. When using a full common carotid ligature, one must be aware that the extent and nature of the ensuing remodeling response will be directly dependent upon the precise site of tissue harvest in relation to the exact site of ligature placement. In other words, if the ligature is placed immediately proximal to the internal/external bifurcation to cease all common carotid arterial flow, then the more proximal one moves along the length of the common carotid artery, the less robust the remodeling response becomes. This is due to reducing levels of flow stagnation the further one 


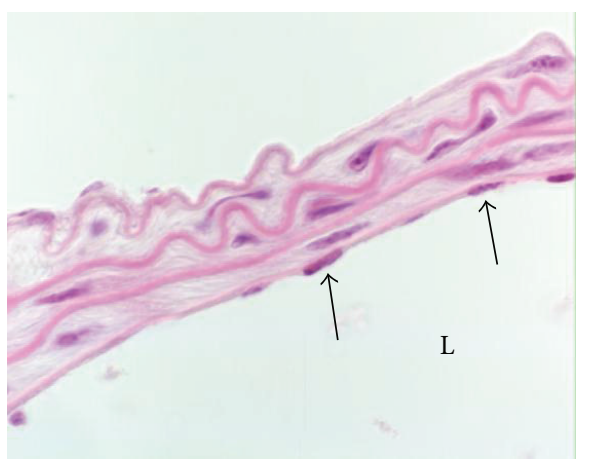

(a)

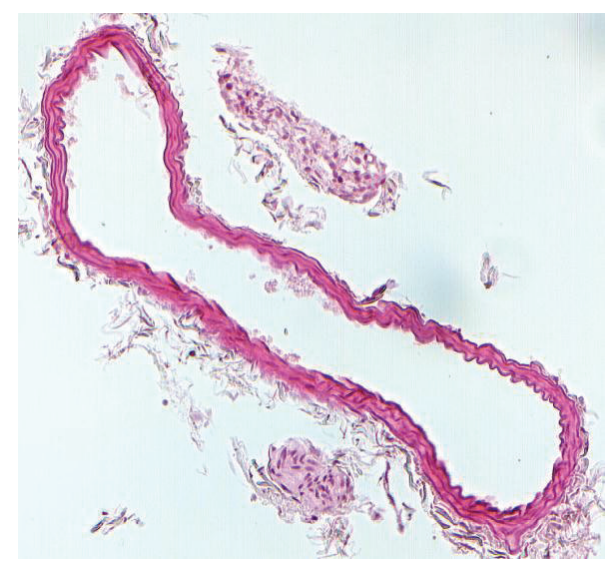

(c)

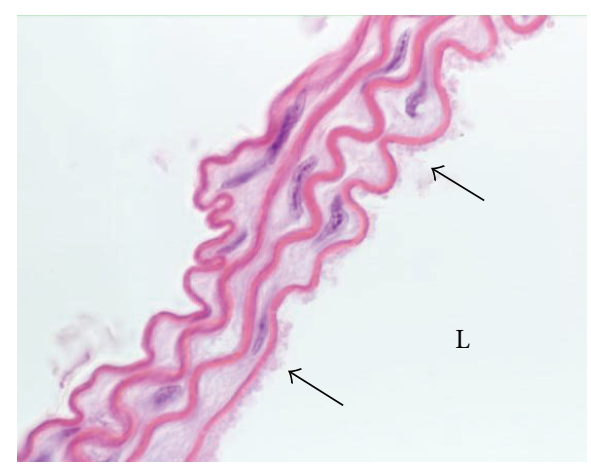

(b)

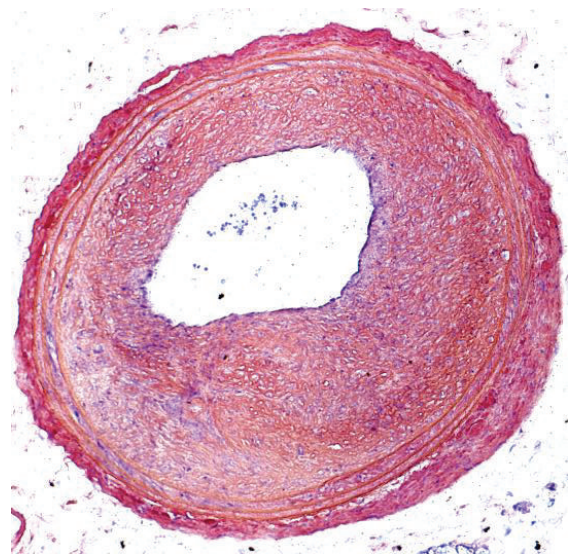

(d)

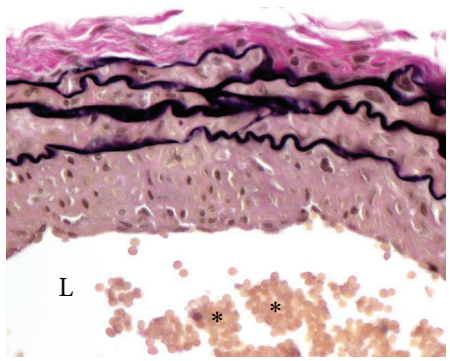

(f)

(e)

FIGURE 3: Representative mouse carotid artery cross-sections with/without carotid artery wire denudation. (a): a hematoxylin and eosin (H\&E)-stained high magnification image of an uninjured carotid artery with nuclear staining of intimal endothelial cells (arrows) and a clear patent lumen (L). (b): a H\&E-stained high magnification image of a mouse carotid artery 30 minutes post-wire denudation injury. A platelet-rich monolayer covering the intimal lining is clearly evident (arrows). (c): a H\&E-stained cross-section of a mouse uninjured carotid artery and (d): a cross-section of a wire-injured carotid artery 4 weeks post-injury showing a robust and concentric neointima with severe luminal obstruction. (e): a high magnification Verhoeff-Van Gieson-stained cross-section of a sham-operated (without wire denudation) mouse carotid artery 4 weeks post-sham surgery with a clear lumen and a thin intimal lining. (f): a mouse Verhoeff-Van Gieson-stained carotid artery cross-section 4 weeks post-wire injury with significant neointima and a partially thrombosed, platelet-rich lumen (denoted by $*$ ).

moves away from the ligature site. This caveat is apparent in the photomicrograph examples shown in Figure 4 which depict cross-sections from mouse carotid arteries following full flow obstruction via a common carotid artery ligation. Figure 4(a) shows an uninjured artery while Figures 4(b) through 4(e) show artery cross-sections 4 weeks after the ligation injury with tissues derived from the same mouse; Figure 4(b) was obtained at $10 \mu \mathrm{m}$ proximal to the site of ligation on the common carotid; Figure 4(c) was obtained at $100 \mu \mathrm{m}$ proximally; Figure $4(\mathrm{~d})$ was obtained at $300 \mu \mathrm{m}$, and Figure 4(e) at $400 \mu \mathrm{m}$ proximally. It is readily observed that the degree of neointimal development and the severity of stenosis is reduced the more distance one moves away from the site of ligature.

\section{Discussion}

Clinical inference of basic science research findings often necessitates the use of in vivo models to test and validate in vitro results. Additionally, in the whole body environment 


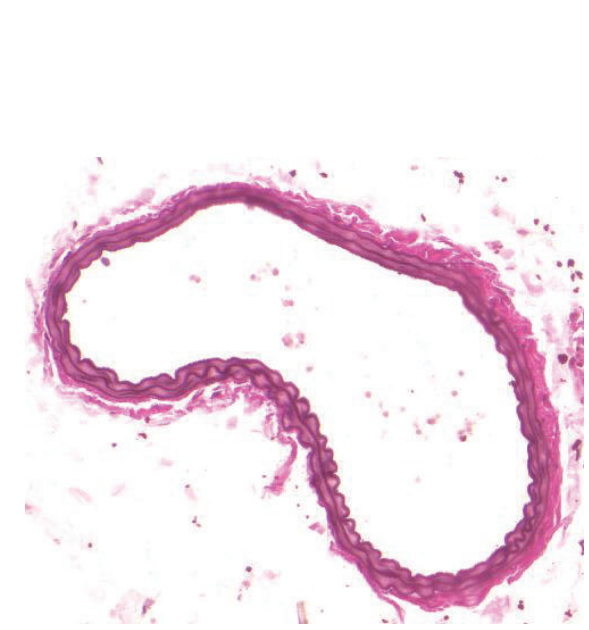

(a)

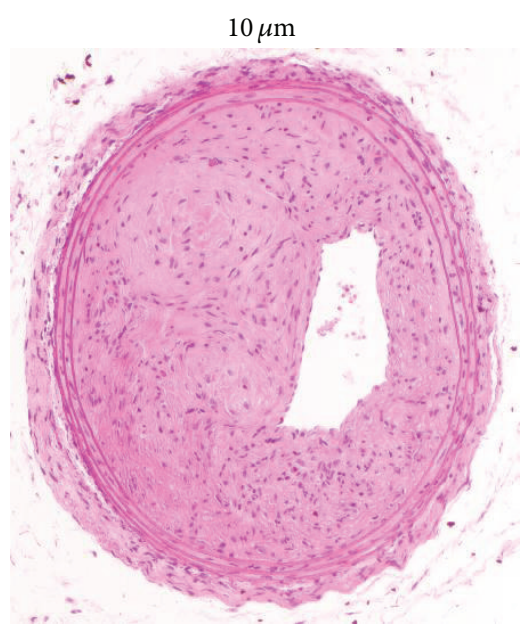

(b)

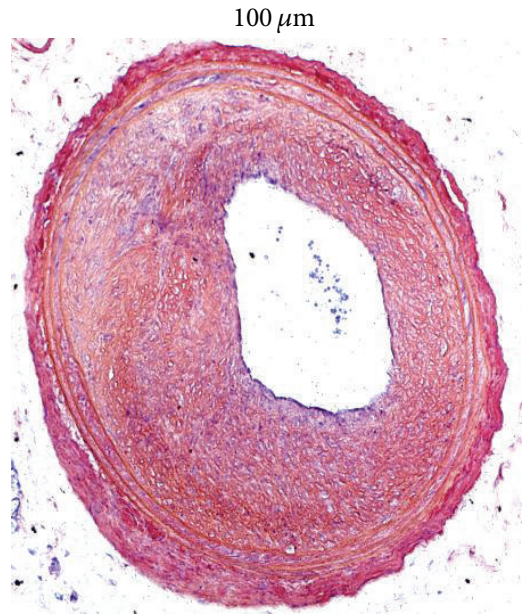

(c)

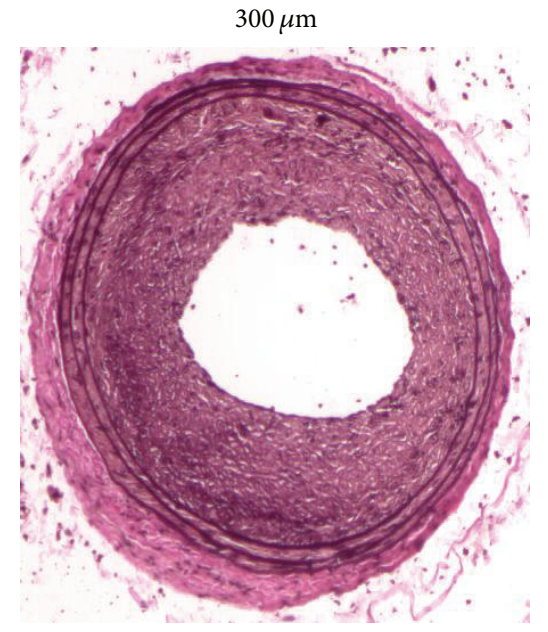

(d)

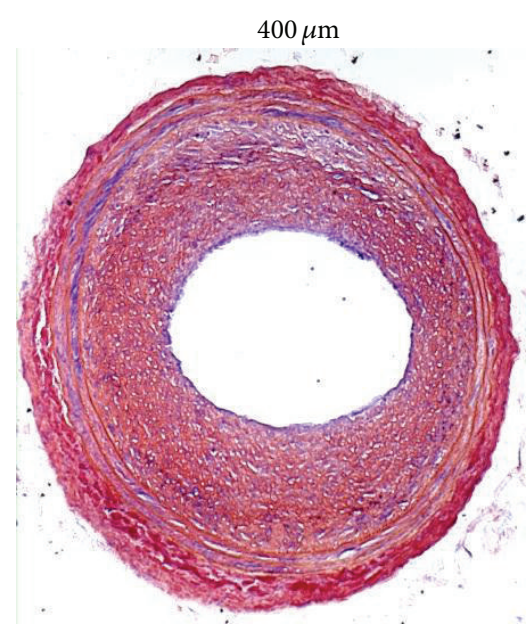

(e)

FIGURE 4: Photomicrographs of mouse carotid artery cross-sections following complete blood flow obstruction via a common carotid artery ligation. (a): an uninjured artery with clear lumen. ((b)-(e)): carotid artery cross-sections obtained 4 weeks following common carotid ligation from the same mouse. Vessel shown in (b) was obtained $10 \mu \mathrm{m}$ proximal to the site of ligation, (c) was obtained $100 \mu \mathrm{m}$ proximal, (d) $300 \mu \mathrm{m}$ and (e) $400 \mu \mathrm{m}$ proximal to the site of ligation. It is noted that the degree of neointimal formation and the severity of stenosis is reduced the more proximal one moves away from the site of ligature.

input from associated elements such as diverse resident cells, circulating cells including progenitor stem cells and inflammatory mediators and systemic factors including endocrine, hormonal and neural signals are considered that cannot realistically be found in controlled in vitro isolated cell or tissue environments. Thus, in vivo proof-of-concept approaches are critically essential and clinically necessary for the translation of benchtop science to the human condition. The in vivo models described herein constitute established approaches to examine the in vivo responses to vascular injury and ensuing growth and remodeling processes and thus serve as highly valuable experimental tools.

Access to the common carotid artery and its branches and experimental perturbations via medial wall distension, endothelial denudation, and/or hemodynamic changes facilitates the study of numerous growth and remodeling events in the intact system. Successfully isolating this area of the vasculature exposes the common carotid and the internal/external bifurcation and allows researchers to execute a multitude of advanced techniques including balloon injury, endothelial denudation, partial or complete flow obstruction, or adventitial cuff placement. Though these models are well-studied, the methodologies for swiftly, safely, and accurately accessing the left common carotid artery and detailed protocols of these experimental models are not sufficiently characterized in the literature. Therefore, in this report we provide a detailed discussion of these models and surgical protocols with emphasis on the initial access to and subsequent experimental intervention of the carotid artery vasculature.

The rat carotid artery balloon injury model is perhaps the best characterized and most commonly used rodent model for vascular growth and remodeling studies. Methodologically consisting of carotid artery access and isolation 
of the carotid artery vasculature followed by insertion of a balloon embolectomy catheter through an external branch arteriotomy, this approach entails catheter-induced endothelial denudation and medial wall expansion. The adaptive response to this injury involves vascular cell dedifferentiation into synthetic phenotypes and is typified by VSM cellular replication and migration, VSM cell apoptosis and necrosis, enhanced synthesis and deposition of extracellular matrix (ECM) components, vascular endothelial regeneration from the border zones, luminal stenosis, and establishment of a neointima in time-dependent fashion. Vascular cell synthesis normally proceeds until the intimal lining along the entire length of the denuded region is reendothelialized and restored. Thus, this model can be used to examine both vascular cell proliferation and growth and gross vessel wall remodeling along with endothelial cell regeneration and intimal reestablishment.

Several caveats exist, though, regarding the rat carotid artery balloon injury model that require brief discussion. Although a primary emphasis of research being performed in our laboratory is the role of VSM in mediating growth and remodeling responses including the rat balloon injury model $[5,6,14,16-25]$, in diseased human vasculature, a mixed population of VSM cells and vascular endothelial cells, macrophages and other inflammatory cells including Tcells, and circulating progenitors such as stem cells interact to regulate the growth response to inimical stimuli. This multifactorial nature of the response to disease and/or injury is a positive attribute of the rat model whereby an investigator can examine these disparate cell types in a whole body setting, either independently or in concert with the full in vivo milieu. In addition, anatomical constraints of rat vasculature compared to vasculature in other species including humans consist of a lower percentage of medial wall elastin, a condensed subintimal layer, and a lack of a significant vasa vasorum [26]. Regarding the experimental protocol, the degree of balloon inflation will directly determine extent of vascular injury and the ensuing growth response as well [27]. Thus, consistency and reproducibility of balloon inflation within and between animals in any particular cohort within any particular study are essential and must be maintained. Despite these potential limitations, the rat balloon injury model is a valuable tool to study diverse growth-regulatory mechanisms under in vivo conditions.

The arterial wire denudation injury model also represents a valuable tool to examine growth-specific indices in the whole body. As mentioned, methodologically, this approach consists of passing an angiocatheter guide wire through the common carotid artery to remove the intimal endothelial lining in the absence of medial wall expansion. Rapid platelet adherence to the subendothelial matrix and VSM and endothelial cell proliferation and migration in the presence of patent arterial flow characterize the response, which results in formation of a concentric neointima between 2 and 4 weeks after the injury. Similar to the rat model, this approach allows analyses of both VSM and vascular endothelial cell growth responses. Importantly, this as well as the mouse vascular ligation models described below makes use of genetic tools that have been developed over the years. A vast number of transgenic mouse models have been developed for a wide array of growth-regulatory factors that allow non-pharmacologic means to ablate specific elements in the growth response. These tools circumvent potential offtarget and specificity/selectivity concerns regarding pharmacotherapy while utilizing global and/or tissue-specific, even conditional, ablation of specific elements, thus representing significant advancement over previously used approaches. Regarding the ligation models, a unique appeal for these approaches is that they have the ability to modify flowdependent variables (shear stress, turbulent versus laminar flow, etc.) in the presence of an unperturbed vessel wall. Depending on the exact site of ligature placement, these models also allow varying degrees of flow modulation in vivo and can be used to complement in vitro flow viscometry or other flow-modifying approaches.

A general caveat of the rat and mouse models is that, unless previously established in a priori fashion via diet, pharmacology or choice of a genetic model is usually performed on otherwise normal eutrophic blood vessels that lack preexisting atherogenic or vasoproliferative pathologies. This is in contrast to clinical balloon angioplasty or other luminal interventional or endarterectomy procedures performed on diseased vasculature in humans. Although the response of healthy vessels to vascular intervention involves many of the same cellular, biochemical, and molecular signals that are involved during vascular pathogenesis, these are independent processes and should not be confused. Anatomically, variation can exist in the exact location of the common carotid artery bifurcation. In most animals, the branch point for the internal and external carotid arteries occurs on the distal common carotid at a site that provides straightforward access to the external branch for surgical intervention; however, in some animals the bifurcation occurs more cephalically, thus making a shorter segment on the external branch for vascular access. Indeed, interanimal variation not only in the response to surgical intervention but also in the exact geometry of the carotid vasculature must be considered.

Although not the focus of this paper, one should also keep in mind that alternate carotid artery injury models exist that could also serve as complementary proof-of-concept in vivo approaches specific to the nature of the study. These include a perivascular adventitial cuff method [28-30], perivascular electrical shock [31], or partial flow obstruction through ligation of the internal, external, and/or occipital branches [15]. Indeed, among these various animal models, investigators must choose carefully the most appropriate and relevant approach to replicate their in vitro findings.

\section{Conclusions}

In summary, herein we characterize the rat carotid artery balloon injury model, the mouse carotid artery wire denudation model, and rat and mouse carotid artery ligation models that have the capacity of translating in vitro findings to the whole animal. These interventional models require surgical vascular access and manipulation of the left common carotid artery and associated vasculature and provide researchers with the ability to study various cellular and molecular pathways that 
can be anatomically related to events that occur in the human condition. Thus, these experimental models represent practically and clinically significant in vivo approaches that can be used to complement and validate in vitro findings in a whole body setting with the hopes of recapitulating many of the biophysical, cellular, and molecular mechanisms elemental in vascular growth responses. Indeed, these excellent proofof-concept approaches are essential to our understanding of basic science findings and their true translational and clinical significance.

\section{Acknowledgments}

This project was supported by the National Institutes of Health, National Heart, Lung, and Blood Institute Award no. HL-81720. This content is solely the responsibility of the authors and does not necessarily represent the official views of the NHLBI or the NIH.

\section{References}

[1] A. W. Clowes, M. A. Reidy, and M. M. Clowes, "Mechanisms of stenosis after arterial injury," Laboratory Investigation, vol. 49, no. 2, pp. 208-215, 1983 .

[2] A. W. Clowes, M. A. Reidy, and M. M. Clowes, "Kinetics of cellular proliferation after arterial injury. I. Smooth muscle growth in the absence of endothelium," Laboratory Investigation, vol. 49, no. 3, pp. 327-333, 1983.

[3] A. W. Clowes and M. M. Clowes, "Kinetics of cellular proliferation after arterial injury. II. Inhibition of smooth muscle growth by heparin," Laboratory Investigation, vol. 52, no. 6, pp. 611-616, 1985.

[4] A. W. Clowes, M. M. Clowes, and M. A. Reidy, "Kinetics of cellular proliferation after arterial injury. III. Endothelial and smooth muscle growth in chronically denuded vessels," Laboratory Investigation, vol. 54, no. 3, pp. 295-303, 1986.

[5] D. A. Tulis, "Rat carotid artery balloon injury model," Methods in molecular medicine, vol. 139, pp. 31-66, 2007.

[6] D. A. Tulis, "Histological and morphometric analyses for rat carotid balloon injury model," Methods in molecular medicine, vol. 139, pp. 31-66, 2007.

[7] V. Lindner, J. Fingerle, and M. A. Reidy, "Mouse model of arterial injury," Circulation Research, vol. 73, no. 5, pp. 792-796, 1993.

[8] T. R. Sullivan Jr., R. H. Karas, M. Aronovitz et al., "Estrogen inhibits the response-to-injury in a mouse carotid artery model," Journal of Clinical Investigation, vol. 96, no. 5, pp. 24822488, 1995.

[9] M. D. Iafrati, R. H. Karas, M. Aronovitz et al., "Estrogen inhibits the vascular injury response in estrogen receptor $\alpha$-deficient mice," Nature Medicine, vol. 3, no. 5, pp. 545-548, 1997.

[10] A. Kumar and V. Lindner, "Remodeling with neointima formation in the mouse carotid artery after cessation of blood flow," Arteriosclerosis, Thrombosis, and Vascular Biology, vol. 17, no. 10, pp. 2238-2244, 1997.

[11] Y. Yuan, L. Liao, D. A. Tulis, and J. Xu, "Steroid receptor coactivator-3 is required for inhibition of neointima formation by estrogen," Circulation, vol. 105, no. 22, pp. 2653-2659, 2002.

[12] B. L. Langille and F. O'Donnell, "Reductions in arterial diameter produced by chronic decreases in blood flow are endotheliumdependent," Science, vol. 231, no. 4736, pp. 405-407, 1986.
[13] B. L. Langille, M. P. Bendeck, and F. W. Keeley, "Adaptations of carotid arteries of young and mature rabbits to reduced carotid blood flow," American Journal of Physiology, vol. 256, no. 4, pp. H931-H939, 1989.

[14] X. Yang, D. P. Thomas, X. Zhang et al., "Curcumin inhibits platelet-derived growth factor-stimulated vascular smooth muscle cell function and injury-induced neointima formation," Arteriosclerosis, Thrombosis, and Vascular Biology, vol. 26, no. 1, pp. 85-90, 2006.

[15] D. Nam, C. W. Ni, A. Rezvan et al., "Partial carotid ligation is a model of acutely induced disturbed flow, leading to rapid endothelial dysfunction and atherosclerosis," American Journal of Physiology, vol. 297, no. 4, pp. H1535-H1543, 2009.

[16] C. N. Joshi, D. N. Martin, J. C. Fox, N. N. Mendelev, T. A. Brown, and D. A. Tulis, "The soluble guanylate cyclase stimulator BAY 41-2272 inhibits vascular smooth muscle growth through the cAMP-dependent protein kinase and cGMP-dependent protein kinase pathways," Journal of Pharmacology and Experimental Therapeutics, vol. 339, pp. 394-402, 2011.

[17] D. A. Tulis, W. Durante, K. J. Peyton, A. J. Evans, and A. I. Schafer, "Heme oxygenase-1 attenuates vascular remodeling following balloon injury in rat carotid arteries," Atherosclerosis, vol. 155, no. 1, pp. 113-122, 2001.

[18] D. A. Tulis, W. Durante, X. Liu, A. J. Evans, K. J. Peyton, and A. I. Schafer, "Adenovirus-mediated heme oxygenase-1 gene delivery inhibits injury-induced vascular neointima formation," Circulation, vol. 104, no. 22, pp. 2710-2715, 2001.

[19] D. A. Tulis, W. Durante, K. J. Peyton, G. B. Chapman, A. J. Evans, and A. I. Schafer, "YC-1, a benzyl indazole derivative, stimulates vascular cGMP and inhibits neointima formation," Biochemical and Biophysical Research Communications, vol. 279, no. 2, pp. 646-652, 2000.

[20] D. A. Tulis, K. S. Bohl Masters, E. A. Lipke et al., "YC-1-mediated vascular protection through inhibition of smooth muscle cell proliferation and platelet function," Biochemical and Biophysical Research Communications, vol. 291, no. 4, pp. 1014-1021, 2002.

[21] A. N. Keswani, K. J. Peyton, W. Durante, A. I. Schafer, and D. A. Tulis, "The cyclic GMP modulators YC-1 and zaprinast reduce vessel remodeling through antiproliferative and proapoptotic effects," Journal of Cardiovascular Pharmacology and Therapeutics, vol. 14, no. 2, pp. 116-124, 2009.

[22] D. A. Tulis, Z. H. Mnjoyan, R. L. Schiesser et al., "Adenoviral gene transfer of fortilin attenuates neointima formation through suppression of vascular smooth muscle cell proliferation and migration," Circulation, vol. 107, no. 1, pp. 98-105, 2003.

[23] K. J. Peyton, D. Ensenat, M. A. Azam et al., "Arginase promotes neointima formation in rat injured carotid arteries," Arteriosclerosis, Thrombosis, and Vascular Biology, vol. 29, no. 4, pp. 488494, 2009.

[24] K. J. Peyton, A. R. Shebib, M. A. Azam, X. Liu, D. A. Tulis, and W. Durante, "Bilirubin inhibits neointima formation and vascular smooth muscle cell proliferation and migration," Frontiers in Pharmacology, vol. 3, article 48, 2012.

[25] J. D. Stone, A. Narine, P. R. Shaver, J. C. Fox, J. R. Vuncannon, and D. A. Tulis, "AMP-activated protein kinase inhibits vascular smooth muscle cell proliferation and migration and vascular remodeling following injury," American Journal of Physiology, vol. 304, pp. H369-H381, 2013.

[26] F. H. Sims, "A comparison of structural features of the walls of coronary arteries from 10 different species," Pathology, vol. 21, no. 2, pp. 115-124, 1989. 
[27] C. Indolfi, G. Esposito, E. Di Lorenzo et al., "Smooth muscle cell proliferation is proportional to the degree of balloon injury in a rat model of angioplasty," Circulation, vol. 92, no. 5, pp. 12301235, 1995.

[28] D. W. Courtman, A. Cho, L. Langille, and G. J. Wilson, "Eliminating arterial pulsatile strain by external banding induces medial but not neointimal atrophy and apoptosis in the rabbit," American Journal of Pathology, vol. 153, no. 6, pp. 1723-1729, 1998.

[29] R. F. G. Booth, J. F. Martin, A. C. Honey, D. G. Hassall, J. E. Beesley, and S. Moncada, "Rapid development of atherosclerotic lesions in the rabbit carotid artery induced by perivascular manipulation," Atherosclerosis, vol. 76, no. 2-3, pp. 257-268, 1989.

[30] M. T. Kuhlmann, S. Cuhlmann, I. Hoppe et al., "Implantation of a carotid cuff for triggering shear-stress induced atherosclerosis in mice," Atherosclerosis, vol. 223, pp. 314-320, 2012.

[31] P. Carmeliet, L. Moons, J. M. Stassen et al., "Vascular wound healing and neointima formation induced by perivascular electric injury in mice," American Journal of Pathology, vol. 150, no. 2, pp. 761-776, 1997. 


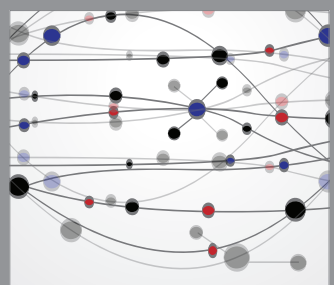

The Scientific World Journal
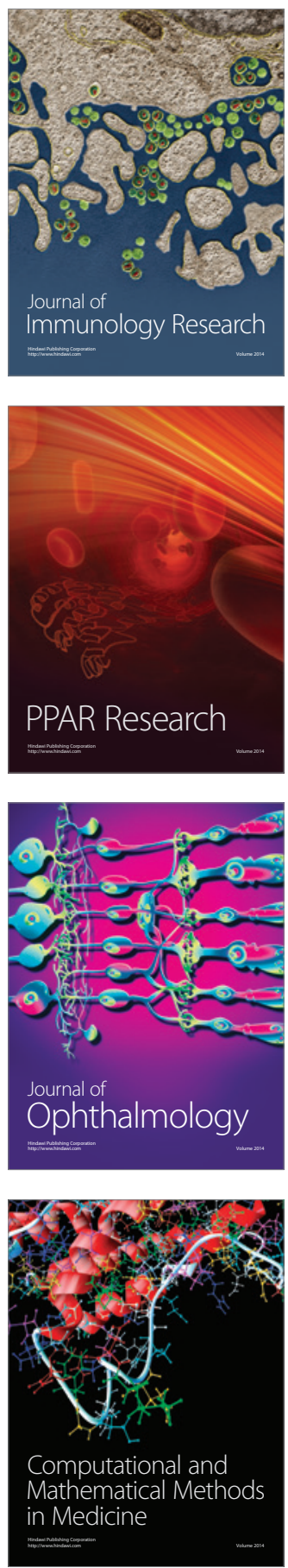

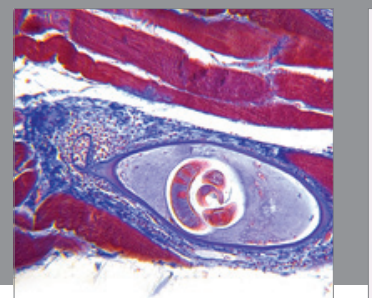

Gastroenterology

Research and Practice
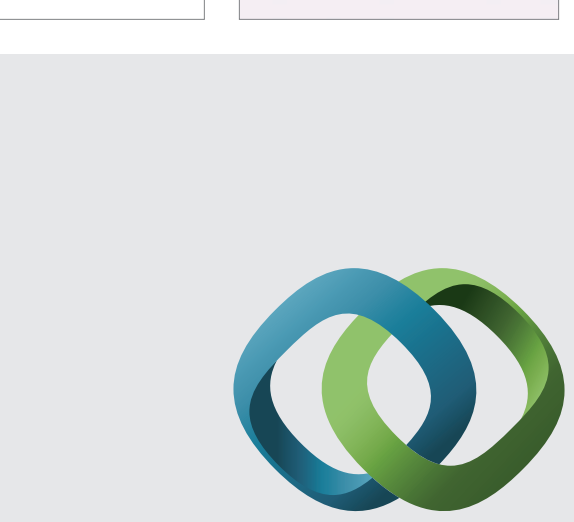

\section{Hindawi}

Submit your manuscripts at

http://www.hindawi.com
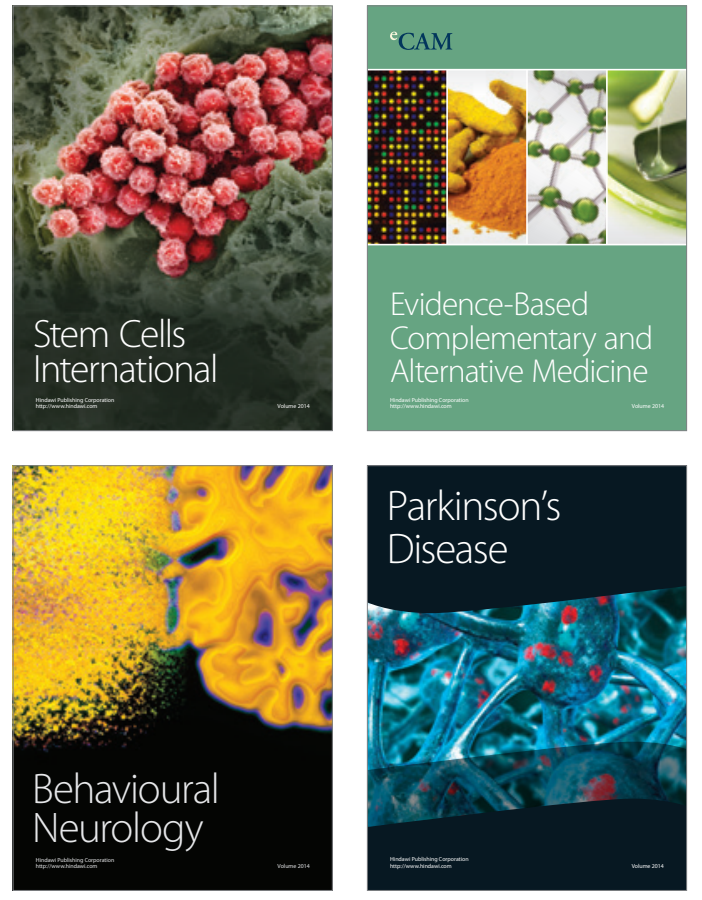
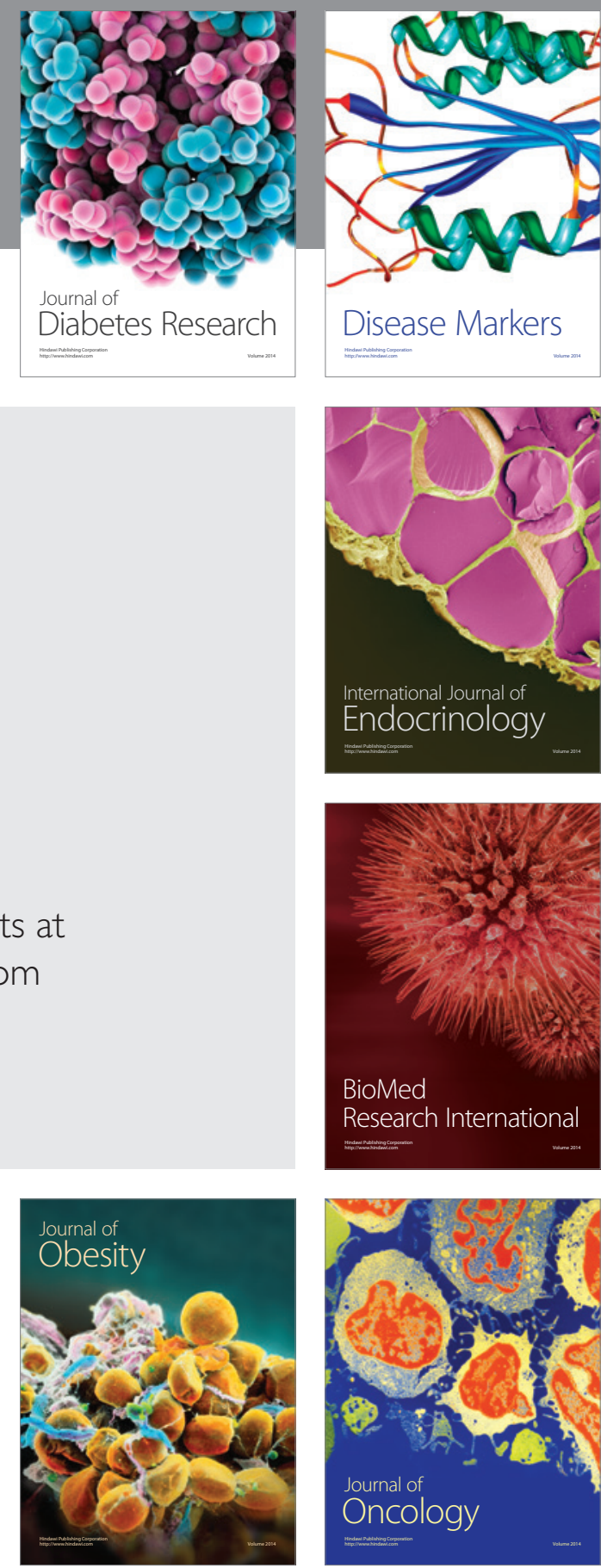

Disease Markers
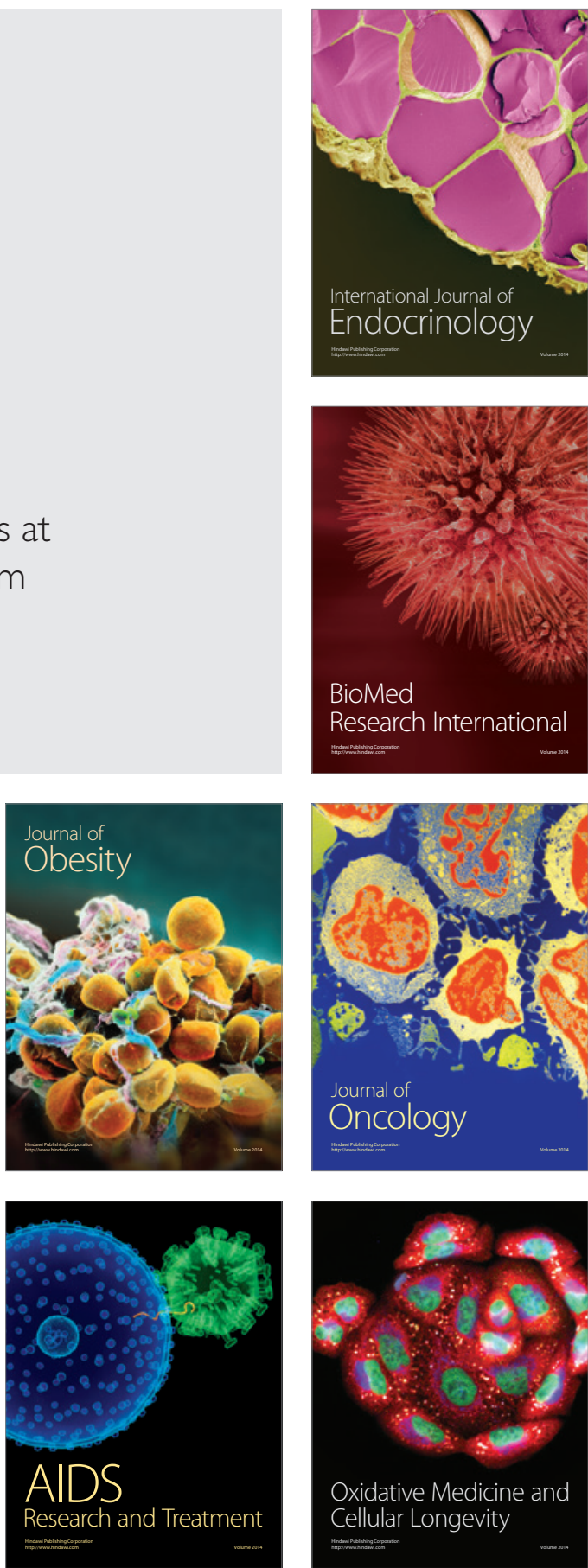